\title{
An evaluation of 1 year of advice calls to a tropical and infectious disease referral Centre
}

\author{
Authors: Tom Wingfield, ${ }^{\mathrm{A}}$ Mike B] Beadsworth, ${ }^{\mathrm{B}}$ Nicholas ] Beeching, ${ }^{\mathrm{C}}$ Susan Gould, ${ }^{\mathrm{D}}$ Luke Mair ${ }^{\mathrm{D}}$ and \\ Emmanuel Nsutebu ${ }^{\mathrm{E}}$
}

\section{Objectives}

Many secondary care departments receive external advice calls. However, systematic advice-call documentation is uncommon and evidence on call nature and burden infrequent. The Liverpool tropical and infectious disease unit (TIDU) provides specialist advice locally, regionally and nationally. We created and evaluated a recording system to document advice calls received by TIDU.

\section{Methods}

An electronic advice-call recording system was created for TIDU specialist trainees to document complex, predominantly external calls. Fourteen months of advice calls were summarised, analysed and recommendations for other departments wishing to replicate this system made.

Results

Five-hundred and ninety calls regarding 362 patients were documented. Median patient age was 44 years (interquartile range 29-56 years) and 56\% were male. Sixty-nine per cent of patients discussed were referred from secondary healthcare, half from emergency or acute medicine departments; $43 \%$ of patients were returning travellers; $59 \%$ of returning travellers had undifferentiated fever, one-third of whom returned from sub-Saharan Africa; $32 \%$ of patients discussed were further reviewed at TIDU. Interim 6-month review showed good user acceptability of the system.

Authors: A senior clinical lecturer and honorary consultant physician in infectious diseases, Liverpool School of Tropical Medicine, Liverpool, UK, The Royal Liverpool and Broadgreen University Hospitals NHS Trust, Liverpool, UK and Karolinska Institutet, Stockholm, Sweden; Bhonorary teaching fellow and clinical director, Liverpool School of Tropical Medicine, Liverpool, UK and The Royal Liverpool and Broadgreen University Hospitals NHS Trust,

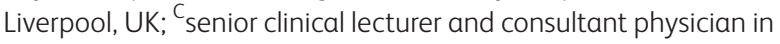
infectious diseases, Liverpool School of Tropical Medicine, Liverpool, UK and The Royal Liverpool and Broadgreen University Hospitals NHS Trust, Liverpool, UK; ${ }^{D}$ specialist trainee, The Royal Liverpool and Broadgreen University Hospitals NHS Trust, Liverpool, UK; ${ }^{E_{C}}$ consultant physician in infectious diseases, The Royal Liverpool and Broadgreen University Hospitals NHS Trust, Liverpool, UK

\begin{abstract}
Conclusions
Implementing an advice-call recording system was feasible within TIDU. Call and follow-up burden was high with advice regarding fever in returned travellers predominating. Similar systems could improve clinical governance, patient care and service delivery in other secondary care departments.
\end{abstract}

KEYWORDS: Travel medicine, infectious and tropical diseases, advice-calls documentation, quality improvement project

DOI: 10.7861/clinmed.2019-0201

\section{Background}

Many medical departments in NHS hospitals receive regular advice calls from general practitioners or teams at external hospitals to discuss patients' diagnosis and care. There is little evidence to suggest that there are formal systems in place to document the number and nature of such calls and the outcomes of the patients involved. Therefore, the burden on staff costs and time of dealing with such calls remains unknown and the continuity of the advice given is unclear. In conjunction with the Liverpool School of Tropical Medicine (LSTM), the tropical and infectious disease unit (TIDU) at Liverpool University Hospitals NHS Foundation Trust (LUFT) provides specialist advice locally, regionally and nationally in the UK. This service is separate from calls received at LSTM, referrals from LUFT's emergency department, TIDU's combined infectious diseases and microbiology consult service within LUFT, or coverage provided to the national imported fever service. The initial point of contact for TIDU specialist advice is a TIDU specialist trainee through the on-call telephone, to which trainees are allocated by rota throughout the year, with evening and weekend on-call duties changing hands on a daily basis.

Until June 2017, no central system for documentation of advice calls existed. TIDU specialist trainees receiving advice calls would document their own call notes, which were inaccessible to other trainees and/or the multidisciplinary team (MDT). Repeated advice calls regarding the same patient (eg from a general practitioner or secondary healthcare) were often received by different trainees. Most of these calls concerned external patients who were not registered in the local trust electronic record system, which could not therefore be used for documentation. TIDU specialist trainees reported that this lack of robust documentation led to inadequate 
handover and discontinuity of patient care. Moreover, the lack of extant data regarding call volumes, clinical advice given, communicable disease recognition, patient outcomes, costs and remuneration of such services, was perceived to overlook a substantial workload and thus be a barrier to workforce planning and resource allocation. Therefore, it was decided departmentally that an advice-call recording system and database for such predominantly external calls should be created and evaluated.

The aims of the quality improvement project (QIP) were to centralise information from clinical referrals for advice and/or transfer from external hospitals, primary healthcare facilities or other healthcare providers; improve continuity of virtual care and advice from TIDU for people not currently inpatients within TIDU; improve documentation in line with good clinical and medicolegal practice and accountability; create a database for calls taken which would be useful for audit purposes and reviewing TIDU service provision; and provide a practical description of replicable methods useful to support other general and specialist units to implement similar systems. We describe the design and implementation of this advice-call recording system and present the findings from analysis of data collected in the first 14 months. Finally, we use our results and experience to generate practical recommendations to support both general and specialist medical departments to set up similar, locally-adapted systems.

\section{Methods}

In May 2017, an electronic advice-call template was created (Box 1) to be used by TIDU specialist trainees to record complex, external calls. The project was registered with the local QIP department and also with the national online QIP repository, 'Life QI' (see supplementary material S1).

A standard operating procedure (SOP) and criteria for recording calls were developed and agreed (Box 1). The template was filled in as an MS Word document and saved in a shared NHS drive in a secure folder, accessible only by TIDU healthcare professionals. Each new entry was saved in a separate subfolder

Box 1. Standard operating procedure and template for advice-calls documentation

\section{Eligibility criteria}

External call (internal calls are logged on the trust's electronic patient notes system)

Formal diagnosis (infection or non-infection) not yet made

Clinical infection advice given with follow-up / call back requested Complex cases requiring ongoing infection specialist input

Cases being referred to TIDU clinic or listed for transfer to TIDU

\section{Template}

Date of initial or follow-up call

Infection specialist registrar taking call

Referrer name, grade, location and contact details

Patient name, date of birth and NHS identifier

Clinical scenario

Advice given

Infection consultant involved (if appropriate) with a title including the initials and date of birth of the patient discussed. The entries were analysed quarterly by collating and transferring the data into an MS Excel spreadsheet. In June 2017, on implementation, TIDU specialist trainees were trained face-toface and sent an explanatory email about the new system. Further email reminders were sent in August 2017.

In November 2017 and again in June 2018, data were analysed and presented to TIDU specialist trainees and consultants at a local audit and QIP meeting, a satisfaction survey was sent out to obtain user feedback on the system and a departmental email reminder. ${ }^{1}$ In August 2017, 14 months post-implementation, a detailed analysis of all documented advice calls was made describing:

> calls: date of call, total number of calls, number of calls per patient, caller location, caller healthcare service level (eg primary care, secondary care or other), caller grade and caller department

> patient details: age, sex, comorbidities, history of foreign travel, clinical syndrome, whether the patient was known to TIDU (eg had been a previous inpatient or previous/current outpatient under TIDU care) and outcome (eg not discussed again, reviewed by TIDU or reviewed by another specialist team)

> TIDU advice details: advice given concerning investigations and/or treatment, number of TIDU specialist trainees involved per patient episode (eg in some circumstances, multiple calls meant various TIDU specialist trainees gave advice for one patient episode) and whether a TIDU consultant was asked to provided clinical advice about the case. ${ }^{2}$

A priori, the most common clinical syndromes encountered were combined into pragmatic groups: fever in returned traveller, localised infection, pyrexia of unknown origin (PUO), rash, new HIV diagnosis, post-exposure prophylaxis (PEP) for rabies, PEP for needlestick injury, PEP for sexual exposure (PEPSE), neurological syndrome and drug reaction. Full definitions are available from the corresponding author on request.

Operational outcomes of each advice call were divided into three categories: none (eg no further calls or clinical involvement, and clinical outcome not known), TIDU further involvement (eg TIDU specialist care through admission/transfer, outpatient review or MDT opinion) or other hospital/unit further involvement (eg through inpatient consultation, outpatient review, MDT or transfer/admission).

Fourteen months of data on consecutive patients and calls were analysed descriptively by actual number and percentage, and summarised using mean or median averages with range or interquartile range where appropriate. Further exploratory, descriptive analysis using Stata v12 included the comparison of the proportion of advice calls documented that concerned returned travellers in the summer versus the winter months. Z test of proportions was used to identify any differences between seasons with the assumption that there would be no significant difference. No sample size calculations were required and no sampling technique used. The SQUIRE 2.0 guidelines for healthcare quality projects were followed.

This work was a QIP approved by the local audit and quality improvement group at LUFT. The project was also registered with the Life QI platform (www.lifeqisystem.com). All data were anonymised and stored on a secure, password protected TIDU server in line with trust and national data protection and management policies. No ethical approval was sought from other bodies. 


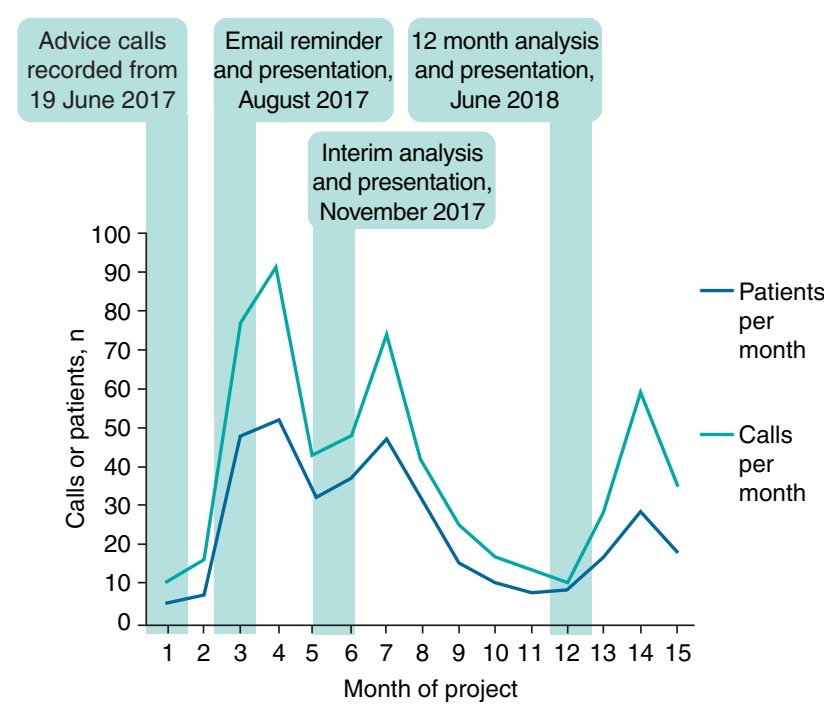

Fig 1. Number of advice calls and patients discussed by month since implementation of advice-calls system.

\section{Results}

Five-hundred and ninety calls concerning 362 patients were documented (mean average 1.6 calls/patient, 10 calls/week); however, the documentation for two patients did not include any sociodemographic or clinical information, or document by whom the call was received, therefore, for the purposes of analysis of sociodemographic and clinical features, $n=360$. The range of calls about a single patient was 1-12 and the range of TIDU specialist trainees involved was one to five. To deal with each call was informally estimated by TIDU specialist trainees to take an average of 10 minutes. The number of documented calls appeared to increase following email reminders and presentations in August 2017, November 2017, and June 2018 (Fig 1). The proportion of all patients discussed who were returning travellers was greater in summer and autumn $(44 \% ; 117 / 265)$ than winter and spring $(33 \%$; 39/125; $p=0.03$; Fig 2).

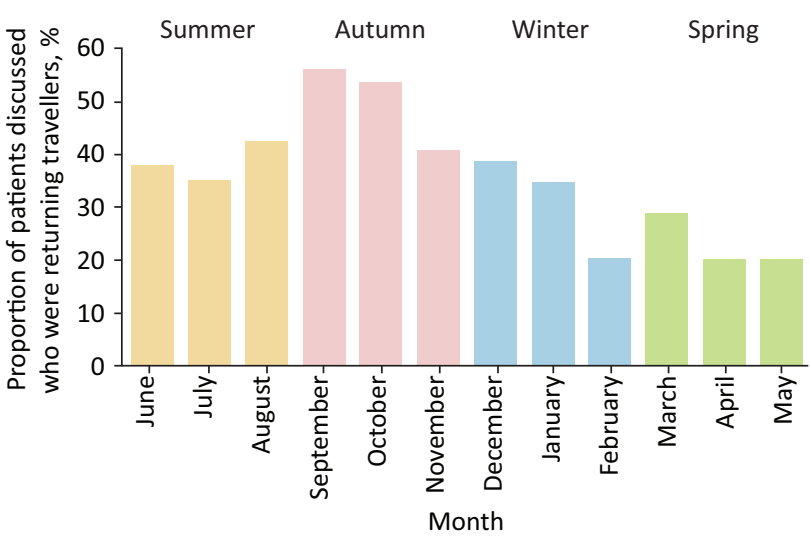

Fig 2. Proportion of patients discussed who were returning travellers by season. Given data from June 2017 to August 2018 were analysed; the proportion of patients discussed who were returning travellers in Summer was a composite of the months of June, July, and August in both 2017 and 2018.
Over two-thirds of patients $(69 \% ; 248 / 360)$ were referred from secondary healthcare (supplementary material S2 - Table 1). Of these, $49 \%(122 / 248)$ were in emergency or acute medicine departments at the time of the initial call. The initial caller was most commonly an internal medicine trainee or equivalent (supplementary material S2 - Table 1).

Supplementary material S3 - Table 2 shows the patient demographic data, clinical syndromes, advice given, outcome overall and returning traveller status. Localised infection (48\%; $171 / 360$ ) was the most common reason for referral; $43 \%$ $(156 / 360)$ of referrals concerned returning travellers, of whom $60 \%(92 / 156)$ had undifferentiated fever and $29 \%(46 / 156)$ had returned from Africa, 23\% (36/156) from South-East Asia and Australasia, 21\% (32/156) from Central Asia and the Indian subcontinent $(21 \%, 32 / 156)$, and $12 \%$ (19/156) from South America and the Caribbean. Advice about patient investigations and treatment was given to the referring team in 92\% (331/360) of cases. TIDU specialist trainees involved TIDU consultants in $51 \%$ (184/360) of cases discussed; $32 \%$ (116/361) of all the cases discussed were subsequently reviewed at TIDU.

Compared with returning travellers, patients with no history of foreign travel were older, more likely to have HIV, hepatitis B and/or C, have non-HIV-related immunosuppression, be a patient known to TIDU, and have either localised infection or pyrexia of unknown origin (supplementary material S3 - Table 2). Returned travellers were more likely to be admitted or transferred to TIDU (supplementary material S3 - Table 2). A post-hoc analysis comparing calls concerning people with HIV versus people without HIV showed no discernible differences in demographic data, clinical syndrome, advice given and patient outcome.

In terms of user satisfaction, 73\% (8/11) TIDU specialist trainees responded to an anonymous online feedback survey. All respondents rated the system's ease of use and support of sound medico-legal documentation as good/excellent, reported that the system was an improvement to the previous standard (eg specialist trainees documenting their own calls with no central, accessible database), and reported that they documented over half of the calls they received. The reasons users gave for not documenting calls received included advice being given and further calls not expected; calls relating to inpatients in the same trust that can be referred online to the TIDU consults team; lack of time; forgetting to document; out-of-hours or overnight calls; and lack of patient or caller information recorded at the time. Respondents made the following recommendations to improve the service: document time spent on calls; estimate admissions avoided and money or time savings to TIDU and TIDU staff; and consider creation of a database that is available from a distance (eg when calls taken at home).

\section{Discussion}

Many secondary care departments, whether specialist or generalist, receive regular advice calls from external primary and other secondary care facilities. In order to give comprehensive advice, especially in rapidly evolving clinical scenarios, a number of calls for virtual consultation concerning the same patient may be made. This can lead to a significant but unquantified burden of clinical work and time. In our busy TIDU referral unit, a simple-to-use advice-call documentation system quantified the previously hidden high burden of calls, demonstrated that a significant number of calls related to returned travellers - especially during the summer 
Box 2. Recommendations for designing and implementing an advice-calls system

> Scoping review: Prior to design and implementation, conduct a scoping exercise and/or staff member diary to elicit volume of calls received, time spent dealing with calls and nature of calls to the department.

MDT involvement: Involve all relevant members of the MDT who receive calls in the design and implementation of the system, and the related SOP.

Q QIP Standards: Register the project with and adhere to the standards of a local and/or national QIP platform.

Practical design: Keep the system design simple and consider how data entered could be extracted and used for audit and QIP purposes (eg use of MS Word or MS Excel platforms against other database software such as MS Access).

> Training and updates: Inform and train users in the department prior to implementation through example scenarios and presentations and ensure regular updates following implementation to maintain system usage and fidelity.

> Monitoring, evaluation and feedback: Perform regular monitoring and evaluation with users - and where possible callers - and consider involvement of colleagues from workforce planning or cost-effectiveness departments to conduct complementary analyses of time and money saved through the call system.

Data review and audit: Aim to systematically collect and review data on the progress and outcomes of patients discussed and entered into the system database.

MDT = multidisciplinary team; QIP = quality improvement project; SOP = standard operating procedure.

months - and showed that a third of calls required further TIDU input. The advice-calls system that we designed and implemented is easily replicable and could be adapted for use in any medical department. In Box 2, we provide the key recommendations for setting up and implementing a similar system.

Evidence relating to clinical advice services is scarce. A limited number of articles describe the role of infection consults in Staphylococcus aureus bacteraemia (SAB) management, a condition with a high case fatality rate. ${ }^{3}$ Two systematic reviews of these studies showed that infection consults improved quality of care through provision of timely, adequate and appropriate anti-staphylococcal antibiotic therapy and also reduced 30-day mortality. ${ }^{4,5}$ In the case of bacteraemia and multidrug resistant enterobacteriaceae bacteraemia, retrospective cohort studies have suggested that an infection review is associated with a reduced risk of mortality. ${ }^{6,7}$ There may also be wider benefits to patients in terms of related preventive therapies such as vaccinations for transplant patients and anti-microbial stewardship. ${ }^{8-10}$

Recent studies have suggested that referrals to infectious diseases specialist teams have doubled over the previous decade in some settings and account for a substantial proportion of all acute admissions; informal ('curbside') consultations in one American infectious diseases unit were estimated to constitute
$17 \%$ of the total unit workload. ${ }^{9,11,12}$ This is important because it has been found that $7 \%$ of infectious diseases diagnoses made by referring non-infection practitioners were incorrect when reviewed by infection physicians, suggesting significant value of timely specialist infection input. ${ }^{10}$ Indeed, pooled evidence from three studies suggests that nearly two-thirds of infection consults result in a change to the referrer's diagnostic and/or therapeutic management plan., ${ }^{9,13-15}$

Evidence regarding the optimal mode of infection review is also limited. Observational studies have suggested that bedside consultation may be superior to telephone consultation in terms of patient outcomes, including SAB-related mortality. ${ }^{10,16}$ However, telephone or electronic consultation can be more time-efficient (with the majority of telephone consultations, as in this study, estimated to be of less than 15 minutes' duration) than bedside or outpatient clinic referrals. ${ }^{11,17,18}$ In addition, implementation studies of telephone advice services for patients within the primary care and secondary general medicine care setting have shown promising results including optimised referral of patients to appropriate healthcare services in Sweden; and reduced need for face-to-face medical consultation and fewer emergency department attendances in the UK..$^{19,20,21}$ Finally, a systematic review showed that an out-of-hours telephone advice-calls service for primary care was an effective form of triage, reduced immediate medical workload and had the potential to reduce overall healthcare costs. ${ }^{22}$ These findings support the need for well-documented telephone advice calls that are complemented by formal, face-to-face (or bedside) review where necessary. Indeed, our findings showed that bedside review occurred in more than a quarter of the cases discussed with our advice-calls service.

Our findings demonstrate that implementation of a simple, userfriendly advice-calls documentation system was feasible and highly acceptable. Such a system could easily be implemented in other departments receiving external advice calls whether generalist or specialist. Indeed, poster presentations of this work have already generated interest and replication of our system and methods within the UK infection community. ${ }^{1,2}$ Documentation of calls appeared to increase following departmental presentations and emails. This finding did not relate to missing data and could suggest compliance issues, lack of sustainability or changing interpretation by specialist trainees as to what merits documentation. However, we interpreted these increases as highlighting the importance of repeated training, reminders and ongoing review throughout the year. Such processes are vital to support the implementation, adaptation and sustainability of similar systems or, indeed, any successful practice changing QIP.

The call workload identified is important given the need to prioritise workload and patient tasks in a busy referral unit that also deals with high consequence infectious diseases. ${ }^{23,24}$ It must be noted that the advice calls documented are an underrepresentation of the total volume of calls received. This is because calls made directly to other members of the MDT (eg consultants, specialist nurses, specialist pharmacists or others) were not documented and a substantial number of calls may not have met eligibility criteria for recording, such as those for telephone consultations that the specialist trainee felt were resolved and unlikely to lead to further calls (eg advice from GPs about testing for Lyme disease following tick exposure in the UK or calls related to side effects of medications). Furthermore, the burden of work identified did not just relate to the calls themselves: 
one-third of patients discussed had further follow-up by TIDU. Our findings are currently being used to support rota and workforce planning, and also resource allocation; the data could also inform recommendations of NHS England specialised commissioning clinical reference group with regard to infectious diseases and/or other specialties.

For patients who were returning travellers, the burden of calls was greatest in the summer and autumn months. These patients were also more likely to be admitted to TIDU. Possible explanations for this increased admission rate include requirement of more extensive acute investigation and management or that less unwell patients were referred to LSTM's tropical on-call phone during work hours. To improve continuity of care and advice given to this patient group, greater resources (eg person-power and/ or rota time) could be allocated to deal with referrals over the summer period, distinct advice streams for returned travellers or those without a history of foreign travel could be developed, and improved regional visibility of methods of referral of patients to TIDU outpatient or MDT services could be implemented.

This project has multiple limitations. Firstly, the calls documented are those made to TIDU and so cannot be generalised to other units or specialties. Nevertheless, implementation and piloting of such a simple advice-call recording system could easily be achieved in different clinical settings. Secondly, the data presented only represent those calls received and documented by TIDU specialist trainees and not the other members of the TIDU's MDT. Future systems could consider monitoring the calls received by all MDT members to gain a more accurate estimate of call burden across an entire unit. Thirdly, the time costs and time burden incurred by such calls were not recorded accurately or prospectively. This would be a useful addition to any QIP aiming to implement a similar system in other units in the future. Finally, it is not possible to infer impact on patient care. Patient and/or caller satisfaction and patient outcomes were not among the primary QIP objectives. This data can be challenging to collect given that the majority of calls involve patients in primary care or external hospitals and would therefore require concerted efforts to follow-up all callers to collate patient outcomes. However, the user feedback on the system was overwhelmingly positive and suggested that, in their subjective opinion, continuity of advice had been improved. Posthoc review of calls concerning two complex patients at external hospitals who required multiple advice calls serve to reinforce this finding. First, over a period of 1 week, we received 11 calls to six members of TIDU staff (four specialist trainees who liaised with two consultants) about an 18-year-old man with suspected hydatid lung disease who was eventually transferred to TIDU, started on treatment and then transferred to a local specialist thoracic surgery team. His diagnosis was confirmed, surgery and treatment were successful, and he made a good recovery. Second, over a period of 10 days, we received nine calls to two specialist trainees about a 50-year-old woman with a new diagnosis of HIV. She had been admitted to an external hospital's critical care unit for severe type 1 respiratory failure due to suspected Pneumocystis jirovecii pneumonia. She was unstable for transfer initially and had a difficult clinical course with a drug reaction to cotrimoxazole but eventually stabilised and was transferred to TIDU. She was stable on anti-retroviral therapy and followed up with TIDU HIV services.

We hope to expand routine collection of patient outcome data going forward and would recommend that other units aiming to implement an advice-call systems do the same. In addition, we would suggest to general medical or other specialist teams that they collect data not only on calls received by physicians but by all members of the MDT in their department.

\section{Conclusions}

In a busy TIDU, the implementation of an advice-call recording system was feasible and well received. In keeping with the role of a national tropical referral centre, returned travellers constituted the majority of advice calls. The previously unquantified burden of calls was higher than anecdotally expected, indicating a substantial proportion of specialist advice for patients external to TIDU. Moreover, one-third of calls led to a direct TIDU review either on an inpatient or outpatient basis. These novel data add to the limited existing literature on advice-call burden, allow an improved understanding of current resource allocation and service development needs in our unit, can contribute to national e-referral systems to any generalist or specialist department, and potentially lead to improved patient care.

\section{Supplementary material}

Additional supplementary material may be found in the online version of this article at www.rcpjournals.org/clinmedicine:

S1 - Advice-calls quality improvement project.

S2 - Table 1. Calls received by referrer location, grade and department over 14 months.

S3 - Table 2. Patient demographic data, clinical syndromes, advice given, outcome overall and returning traveller status.

\section{Funding}

Tom Wingfield is supported by grants from the Wellcome Trust, UK (209075/Z/17/Z); the Academy of Medical Sciences, UK; the Swedish Health Research Council, Sweden; and the National Institute for Health Research (NIHR), UK. Nicholas ] Beeching is based at Liverpool School of Tropical Medicine and receives support from the NIHR Health Protection Research Unit (HPRU) in Emerging and Zoonotic Infections at University of Liverpool in partnership with Public Health England (PHE) in collaboration with Liverpool School of Tropical Medicine. The views expressed here are those of the authors and not necessarily those of the NHS, the NIHR, the Department of Health and Social Care or PHE. This work was conducted independently of the NIHR.

\section{References}

1 Wingfield T, Beeching NJ, Beadsworth M, Nsutebu E. Six months of advice calls to a tropical and infectious diseases referral Centre: a retrospective, descriptive analysis from Liverpool, England. 28th ECCMID: European Conference on Clinical Microbiology and Infectious Diseases 2017:P1297.

2 Wingfield T, Beeching NJ, Beadsworth M, Nsutebu E. One year of advice calls to a tropical and infectious diseases referral Centre: a retrospective, descriptive analysis from Liverpool, England. Federation of Infection Societies Conference 2018 2018:P098.

3 Yilmaz M, Elaldi N, Balkan II et al. Mortality predictors of Staphylococcusaureus bacteremia : a prospective multicenter study. Ann Clin Microbiol Antimicrob 2016;15:7.

4 Vogel M, Schmitz PRH, Hagel S et al. Infectious disease consultation for Staphylococcusaureus bacteremia - A systematic review and meta-analysis. J Infect 2016;72:19-28. 
5 Paulsen J, Solligård E, Damås JK et al. The impact of infectious disease specialist consultation for Staphylococcusaureus bloodstream infections : A systematic review. Open Forum Infect Dis 2016;3:ofw048.

6 Burnham JP, Olsen MA, Stwalley D et al. Infectious diseases consultation reduces 30-day and 1-year all-cause mortality for multidrug-resistant organism infections. Open Forum Infect Dis 2018;5:ofy026

7 Tang G, Huang L, Zong Z. Impact of infectious disease consultation on clinical management and outcome of patients with bloodstream infection: a retrospective cohort study. Sci Rep 2017;712898.

8 Kasper AK, Pallotta AM, Kovacs CS, Spinner ML. Infectious diseases consult improves vaccination adherence in kidney transplant candidates. Vaccine 2018;36:5112-5.

9 Yinnon AM. Whither infectious diseases consultations? Analysis of 14,005 consultations from a 5-year period. Clin Infect Dis 2001;33:1661-7.

10 Petrak RM, Sexton DJ, Butera ML et al. The value of an infectious diseases specialist. Clin Infect Dis 2003;36;1013-7.

11 Kirsh SR, Ho PM, Aron DC. Providing specialty consultant expertise to primary care: An expanding spectrum of modalities. Mayo Clin Proc 2014;89:1416-26.

12 Grace C, Alston WK, Ramundo M et al. The complexity, relative value, and financial worth of curbside consultations in an academic infectious diseases unit. Clin Infect Dis 2010;51;651-5.

13 Rameau C, Mahy S, Simonet Lamm AL et al. Informal consultation at a teaching hospital infectious diseases department. Med Mal Infect 2014;44:107-11.

14 Sexton DJ, Corey GR, Ingram CW, Morris VM, Haywood HB. Consultation in university-based and community-based infectious disease practices: A prospective study. Clin Infect Dis 1995;20: 391-3.

15 Chatterjee A, Lackey SJ. Prospective study of telephone consultation and communication in pediatric infectious diseases. Pediatr Infect Dis ] 2001;20:968-72.
16 Forsblom E, Ruotsalainen E, Ollgren J, Järvinen A. Telephone consultation cannot replace bedside infectious disease consultation in the management of Staphylococcusaureus bacteremia. Clin Infect Dis 2013;56:527-35.

17 Leblebicioglu H, Akbulut A, Ulusoy S et al. Informal consultations in infectious diseases and clinical microbiology practice. Clin Microbiol Infect 2003;9:724-6.

18 Murthy R, Rose G, Liddy C, Afkham A, Keely E. eConsultations to infectious disease specialists: Questions asked and impact on primary care providers' behavior. Open Forum Infect Dis 2017:4:ofx030.

19 Dahlgren K, Holzmann MJ, Carlsson AC et al. The use of a Swedish telephone medical advice service by the elderly-a populationbased study. Scand J Prim Health Care 2017:35:98-104.

20 Lattimer V, George S, Thompson F et al. Safety and effectiveness of nurse telephone consultation in out of hours primary care: Randomised controlled trial. BMJ 1998;317:1054-9.

21 Anderson A, Roland M. Potential for advice from doctors to reduce the number of patients referred to emergency departments by NHS 111 call handlers: Observational study. BMJ Open 2015;5:e009444.

22 Leibowitz R, Day S, Dunt D. A systematic review of the effect of different models of after-hours primary medical care services on clinical outcome, medical workload, and patient and GP satisfaction. Fam Pract 2003;20:311-7.

23 Vaughan A, Aarons E, Astbury ] et al. Two cases of monkeypox imported to the United Kingdom, September 2018. Euro Surveill 2018;23:1800509.

24 Jacobs M, Beadsworth M, Schmid M, Tunbridge A. Provision of care for Ebola. Lancet 2014;384:2105-6.

Address for correspondence: Dr Tom Wingfield, Liverpool School of Tropical Medicine, Pembroke Place, Liverpool L3 5QA, UK.

Email: tom.wingfield@lstmed.ac.uk 